that the disorcer was functional and suggestion that skin sensation would return were succeeded by a prompt renewal of the capacity to detect pin-pricks and some slight return of the capacity to distinguish differences of temperature ; but the complete cutaneous analgesia remained undiminished, and neither deep pin-pricks nor prolonged contact with ice led to pain, discomfort, or change in facial expression.

\section{Method of Investigation}

With the patient lying in bed a series of measurements of the blood pressure were made on the right arm; the left arm (which in common with other parts was devoid of superficial sensation) was placed out of view of the patient. When the blood pressure had reached a steady level a sphygmomanometer cuff placed previously around the upper arm on the left sice was inflated to $170 \mathrm{~mm} . \mathrm{Hg} \rightarrow$ a pressure well above the patient's systolic pressure. -thus completely arresting the circulation through the left arm. For the elicitation of the Hines and Brown blood-pressure-raising reflex small blocks of ice were packed around the patient's left arm, taking care that the operation was inaudible as well as invisible. The general blood pressure was measured again in the right arm while the left arm was being stimulated by the application of ice. To investigate the effect of the blood-pressureraising reflex from muscle the patient opened and closed the left hand with the circulation through the left arm completely arrested until pain of the intermittent claudication type prevented further movement. During the exercise and for some time afterwards measurements of the general blood pressure were made on the right arm.

\section{Results and Discussion}

It was found that after the application of ice to the left arm the blood pressure in the right arm began to rise, although the patient felt no discomfort whatsoever from the ice. The increases in blood pressure averaged $17 \mathrm{~mm}$. $\mathrm{Hg}$ systolic and $11 \mathrm{~mm}$. diastolic. The protocol of one of these investigations is here set out ; three such investigations were made on different days, always with a rise of blood pressure. In order to ensure that the rises of blood pressure were not of psychological origin care was taken to prevent the patient seeing or hearing that anything was being done to the limb. During the investigation the patient's reports of her sensations were either that she felt nothing whatsoever or that something was felt, though not of a painful nature. In the normal person the pain arising from the application of several blocks of ice to the hand and forearm usually becomes aimost intolerable

Protocol showing the Effect upon the Blood Pressure of the Application of Ice to a Limb with Skin Anaesthesia of Hysterical Origin

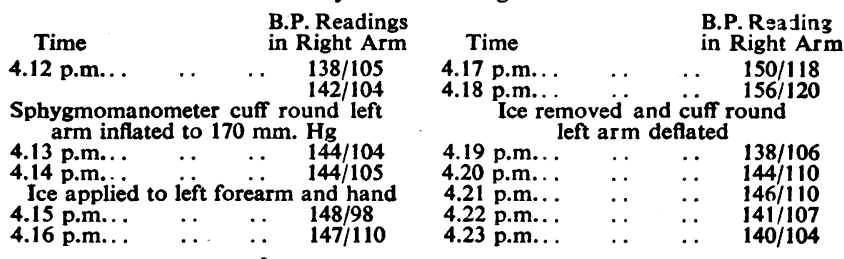

within less than one minute. In this hysterical patient no pain or discomfort whatsoever followed the application of ice to the hand and forearm, yet the normal reflex increase of blood pressure was not abolished by hysterical loss of the patient's capacity to feel this painful stimulus. It was concluded that the rises in blood pressure were of a reflex nature, since, with the circulation of the limb arrested, nerves form the sole channel of communication between the limb and the rest of the body. While eliciting the reflex from muscle it was observed that muscle pain was induced with unusual ease, since twenty-five contractions of the left forearm muscles, effected by opening and closing the left hand during arrest of the circulation in the left arm, gave rise to strong pain of the intermittent claudication type, which was associated, as is usual, with a reflex rise in blood pressure. In a normal person twenty-five strong clenchings of the hand with the forearm ischaemic will not elicit appreciable claudication pain.

Investigations have shown (Alam and Smirk, 1938) that the blood-pressure-raising reflex of Hines and Brown (in which coid is applied to an arm) cannot be elicited from an area in which there is local loss of sensation of organic origin, and it would appear, therefore, that if the observation we have made is of general application the presence or absence of this blood-pressure-raising reflex from skin might provide evidence in doubtful cases as to whether an area of skin anaesthesia was the result of an organic lesion below medullary level or was hysterical in nature. The observation is of further interest in that it demonstrates the integrity of certain nervous pathways in a patient with hysterical skin anaesthesia, and indicates that a similar method of study is applicable to cases in which there is loss of muscle pain.

Should observations such as these be employed in the differential diagnosis of nerve lesions it is of importance to recognize that emotional stimuli also produce striking changes in blood pressure and that the degrees of reflex increase in blood pressure vary considerably, but are seldom less than 10 or more than $50 \mathrm{~mm}$. $\mathbf{H g}$.

\section{Summary}

Observations have been made on a patient with superficial anaesthesia of a hysterical nature, including the skin of the left arm. It was observed that the application of ice to the left arm caused a rise of blood. pressure which was shown to be of reflex origin.

If the patient is unaware of the application of the stimulus a rise of blood pressure on applying cold to an anaesthetic area may be taken to demonstrate the presence of a nervous connexion between the anaesthetic area and the central nervous system, and in some cases would suggest that the anaesthesia is of a hysterical nature.

\section{REFERENCES}

Alam, M., and Smirk, F. H. (1937), J. Physiol., 89, 372.

Aines (1938). Clin. Sci., 3, 24

Hines, E. A., and Brown, G. E. (1932). Proc. Mayo Clin., 7, 332.

\section{TEMPORARY VASCULAR OCCLUSION ENDING FATALLY IN URAEMIA}

\section{BY}

\author{
ALAN M. GLEN, M.B., Ch.B. \\ Senior House-surgeon, County Hospital, Lincoln
}

In view of the recent work on renal failure after compression injuries a case in which temporary occlusion of the vessels to the right leg was followed by uraemia and death seems worth recording.

\section{Case Report}

A workman aged 39 was admitted to hospital shortly after falling some twenty feet through a factory roof on to a concrete floor. He had not been unconscious, was only mildly shocked, and complained of pain chiefly in the right thigh. On examination little was found apart from a contusion over the right greater trochanter and marked limitation of movement of the right hip-joint. He was removed to the ward, and while waiting to be radiographed his condition began to deteriorate rapidly. The routine shock treatment was therefore supplemented by the rapid intravenous administration of two pints of blood plasma, with much benefit - the pulse rate dropping to below 100 a minute and the systolic blood pressure rising to $95 \mathrm{~mm}$. Hg. A third pint was then given by slow drip.

At this time the right lower limb was noticed to be slightly blue and swollen and somewhat colder than its fellow. The inguinal ligament of that sice felt tense and appeared to be pushed up from below. Above it the external iliac vessels 
were pulsating strongly, but below it no impulse could be elicited in the femoral, popliteal, or post-tibial regions. The limb was flexed slightly at the hip-joint, and the foot was raised in the hope that this might relieve the obstruction; but the bluish tinge persisted, the leg grew colder, and cutaneous sensation was gradually lost. The patient complained of desire to pass water and of inability to do so, but was discouraged from making any attempt until damage to the bladder and urethra could be ruled out. A soft rubber catheter was passed into the bladder easily and $5 \mathrm{oz}$. of clear urine withdrawn.

$X$-ray examination revealed a central dislocation of the right hip-joint, with comminution of the floor of the acetabulum, and fractures of both pubic rami of the same side. The head of the femur appeared to be entirely within the cavity of the pelvis. It seemed reasonable, therefore, to assume that the external iliac vessels had either been torn across or been compressed between the fractured superior pubic ramus and the inguinal ligament.

Four hours after admission the external iliac vessels were exposed by extraperitoneal approach uncer local anaesthesia and found to be undamaged and pulsating strongly. Just below this level they were completely obstructed by being forced upwards against the inguinal ligament by the fractured superior pubic ramus. The inguinal ligament was therefore divided at its midpoint and the circulation in the femoral vessels re-established, but almost immediately the patient collapsed. The pulse disappeared at the wrist, his colour became ashen, and his breathing was gasping in character. Pressure was at once reapplied to the released vessels, the head of the table lowered, and 1/2 grain of ephedrine given intramuscularly. In a few minutes the pulse returned at the wrist, his colour improved, and the breathing became quieter. .The vessels were therefore intermittently released for a few seconds at a time and the horizontal position gradually reassumed. A further pint of serum was given, making a total of four pints, and the wound closed. The patient returned to bed in good condition, and overnight received a litre of $5 \%$ glucose saline intravenously.

The following day he appeared well, complaining only of the tingling in his leg, which was a good colour and about the same temperature as its fellow. It had, however, a peculiar feeling of hardness, almost as if the underlying muscles were being held in contraction. The femoral pulse was satisfactory, but no pulse could yet be elicited in the popliteal or ankle regions. Under local anaesthesia a Steinmann pin was passed through the tibial tubercle and an initial traction of $10 \mathrm{lb}$. applied with the hip abducted and slightly flexed. By evening the colour had returned to normal, the posterior tibial artery was pulsating, and cutaneous sensation was beginning to return.

In spite of a fluid intake of over two pints a day by mouth, the amount of urine secreted on the three days following operation was $6 \mathrm{oz}$., nil, and $2 \mathrm{oz}$. respectively. Both samples were acid in reaction and heavily blood-stained. The bowels were somewhat loose, and moved twice daily. There was no evidence of oedema. On the evening of the third day his condition began to deteriorate. He betame drowsy, with intervals of acute and increasing anxiety and short attacks of dyspnoea. The following morning there was occasional retching but no actual vomiting. He was more drowsy, but still had intervals of acute anxiety, and frequently expressed fear of impending death. Examination revealed no evidence of oedema or of pulmonary complications, but a blood-urea test made at 10 a.m. gave a reading of over $200 \mathrm{mg}$. per $100 \mathrm{c} . \mathrm{cm}$., and at midday the patient died, just under four days after the accident.

\section{Post-mortem Examination}

A necropsy was performed two hours after death and the findings are briefly as follows:

The peritoneal cavity contained a small amount of free blood, probably due to two small superficial lacerations of the spleen. The comminuted. fracture of the pelvis was confirmed, and the upper pubic ramus was found thrust against the inguinal ligament with the external iliac vessels arching over it. These vessels were undamaged. The head of the femur was entirely within the pelvic cavity, and there was a fairly extensive retroperitoneal haematoma in the pelvis and extending up the posterior abdominal wall. The muscles of the affected leg appeared normal. The bladder, ureters, and kidneys were un- damaged, though the last-named appeared extremely congested. All other viscera were healthy. Microscopical examination of sections taken from both kidneys revealed gross pathological changes. The glomeruli for the most part seemed normal, though in a few the capsular space contained clear structureless material staining pink with eosin. The tubules, however, showed profound degenerative changes of the lining cells, with pyknotic nuclei and granular degeneration of the cell contents, most pronounced in the proximal tubules. More distally the tubules contained darker-staining reddish-brown material, for the most part coarsely granular but some of it homogeneous, and in a few suggestive of clumped degenerating erythrocytes. The proportion of tubules so affected increased as the pelvis was approached, and in many fields towards the apex of a pyramid over $80 \%$ of the tubules seen appeared to be choked with this granular material.

\section{Summary and Comment}

This is a case of central dislocation of the hip with fracture of the pubic rami of the same side causing obstruction of the external iliac vessels. Relief of the obstruction by dividing the inguinal ligament was followed by sudden and profound collapse comparable to that resulting from the sudden release of a tourniquet left over-long in position.

Death resulted from uraemia within four days of the accident, and the microscopical appearances of the kidneys corresponded closely with those recently described in cases of crush injury (Bywaters and Beall, 1941 ; Beall, Bywaters, Belsey, and Miles, 1941 ; Mayon-White and Solandt, 1941).

The hardness of the limb noticed after operation is interesting in view of the theory recently put forward by Patey and Robertson (1941) that the syndrome may be due to loss of substances from the circulation into the injured limb. Unfortunately no particular attention was paid to this aspect at the time.

It is hoped that the findings described may prove of interest to those investigating such cases and to others who may have to deal with similar injuries.

I wish to thank Mr. G. A. Bagot Waiters for his advice and for permission to publish this case; also Mr. Chandler for the blood chemistry and the preparation of sections.

\section{REFERENCES}

Beall, D., Bywaters, E. G. L., Belsey, R. H. R., and Miles, J. A. R. (1941). British Medical Journal, 1, 432 .

Bywaters, E. G. L., and Beall, D. (1941). Ibid., 1, 427.

Mayon-White, R., and Solandt, O. M. (i941). Ibid., 1, 434

Patey, D. H., and Robertson, J. D. (1941). Lancet, 1, 780.

The province of Sind in North-West India, the population of which is just over four million, has a lower birth rate, death rate. and infant mortality rate than any other Indian province. The latest annual report on public health in Sind gives the birth rate as 20.88, the next lowest being Assam with 29.92; the death rate is 11.55 , the next lowest being the North-West Frontier with 18.74 ; and the infant mortality rate 105 per 1,000 births, as against 130 in the North-West Frontier. For the second year in succession the chief city, Karachi, enjoyed immunity from plague in 1939. What are described as "fevers" accounted for the greatest number of deaths; this category incluc'es many of the deaths due to malaria. Malaria is the disease most prevalent in the rural areas, and is one of the major problems of the province. Its prevalence has been accentuated by the enormous increase in the area brought under cultivation since the opening of the Lloyd barrage. Vigorous anti-malaria measures are being taken, and during the year under review nearly two million 5 -grain tablets of quinine sulphate and four and a half million 2-grain tablets, manufactured at Hyderabad central prison, were supplied for distribution. Next to "fevers," respiratory diseases account for the largest number of deaths. An active anti-tuberculosis campaign is in progress, and clinics in charge of fully trained tuberculosis medical officers are being established in each of the district towns. Cholera was restricted to one village in the Hyderabad district, and only six cases and three ceaths were reported. Small-pox is prevalent and accounted for 565 deaths. Trouble is encountered in administering the vaccination laws. 